\title{
Neurotropic Viral Infections in Bangladesh: Burden and Challenges
}

\author{
Mohammad Enayet Hussain
}

Assistant Professor, Department of Neurology, National Institute of Neurosciences \& Hospital, Dhaka, Bangladesh; Email: enayetdmc@yahoo.com

Neurotropic virus infections continue to cause major disease and economic burdens on society ${ }^{1}$. It poses a major challenge to human health care systems due to the associated morbidity and mortality worldwide. This creates a unique problem in providing treatment to the patients involved. This is largely due to unique features of the central nervous system (CNS), with a plethora of interconnected and interdependent cell types, complex structures and functions, reduced immune surveillance and limited regeneration capacity. Infection by neurotropic viruses as well as the local induced immune responses can irreversibly disrupt the complex structural and functional architecture of the CNS, frequently leaving the patient with a poor or fatal prognosis. Besides immediate and direct effects, there are several neurological disorders often associated with autoimmune mechanisms that are assumed to be delayed virusinduced disorders: multiple sclerosis, GuillainBarré syndrome, narcolepsy and encephalitis lethargica ${ }^{2}$. Neurotropic pathogens can access the brain by various routes including retrograde axonal transport along motor and olfactory neurons, haematogenous spread across the blood-brain barrier (BBB), blood-cerebrospinal fluid barrier, meningeal-cerebrospinal fluid barrier, via direct infection of endothelial cells or via spread of infected leukocytes across the BBB into the brain parenchyma ${ }^{3}$.

Influenza virus infections are the most common viral disease in Bangladesh. Overcrowding is the main reason of this disease burden. The most common extra-respiratory complication of influenza is the development of CNS disease ${ }^{4}$. Influenza virus infections cause some acute CNS diseases. Acute CNS diseases include febrile seizures, acute onset brain dysfunction, meningitis, encephalitis and encephalopathies. In addition, influenza viruses have been linked to the development of Guillan Barré syndrome, Kleine Levin syndrome and transfer myelitis ${ }^{5}$. Maternal influenza has been associated with schizophrenia and bipolar disorder (BD) in the offspring. Acute demyelinating encephalomyelitis (ADME) typically occurs in measles patients. Subacute sclerosing panencephalitis (SSPE) occurs on average 410 years following acute MV infection. Mumps virus was the leading cause of aseptic meningitis in the pre-vaccine era. Pathologically, mumps encephalitis resembles ADME and is characterised by extensive perivascular demyelination in the white matter of the cerebral and cerebellar hemispheres, basal ganglia, midbrain, pons, medulla and spinal cord with areas of demyelination associated with the perivascular infiltration of immune cells, microglia and gliosis. Outbreak of Nipah virus (NiV) has been reported in 2006 in Rajbari district of Bangladesh ${ }^{3}$. Prodromal signs of henipavirus infection are general and include fever, lethargy and headache and occur following an incubation period of 5-14 days. This rapidly progresses to neurological signs of infection including limb weakness and segmental myoclonus. Rabies is common disease in Bangladesh.

It is a neurological disease characterized by fatal encephalitis. In the neurological phase, furious rabies patients may develop paresthesia at the site of exposure, anxiety or agitation, dysautonomia (autonomic dysfunction) including inspiratory spasms, hypersalivation hydrophobia, sometimes in combination with aerophobia and fluctuating mental state. The paralytic form of rabies is rather characterized by progressive weakness and paralysis, with absence of many of the symptoms seen in the neurologic phase of furious rabies. 
Paralytic rabies is often misdiagnosed as GuillainBarré syndrome (GBS), autoimmune disease or stroke.

Chikungunya viral infection is not common in Bangladesh. However, there was an outbreak in 2008 at Rajshahi District caused by Aedes albopictus mosquito commonly presented with high fever, headache, maculopapular rash and painful arthralgia ${ }^{4}$. Furthermore central nervous system involvement can also be found by Chikungunya viral infection like brain swelling, disseminated intravascular coagulation, cerebral or cerebellar hemorrhage, scattered parenchymal petechiae, cerebellar hematoma and hematemesis ${ }^{7}$. In children and adults, neurological manifestations include altered levels of consciousness, cranial nerve deficits, seizures, decreased deep tendon reflexes, psychosis, hemi/paraparesis, paraplegia and involuntary movements. A subset of children of $<1$ year presented with hypotonia, tense fontanelle and status epilepticus ${ }^{8}$. Herpes simplex virus (HSV) infection is the most common cause of infectious encephalitis in humans ${ }^{9}$. However, in Bangladesh Uddin et $\mathrm{al}^{2}$ have reported HSV-2 in a hospital based study and have found that female is more commonly affected than male in young age group though male are affected later. HSV-1 is responsible for up to $90.0 \%$ of HSV encephalitis (HSE) cases, whereas HSV-2 infection being less common and more often presenting clinically as meningitis. Clinically, HSE patients present with headache, fever and alteration of mental status such as confusion, psychosis and also alteration of consciousness from somnolence to stupor and coma. Sequelae are still found and include focal or generalized seizures, personality changes, impaired memory and/or cognition, motor deficits or aphasia. Epstein-Barr virus is the causative agent of infectious mononucleosis (IM) in naïve children and young adults, with clinical symptoms evident 4-6 weeks following infection. Less than $5 \%$ of primary EBV infections cause CNS disease that present as meningitis, encephalitis, cerebellitis, cranial or peripheral neuropathies and polyradiculomyelitis. To prevent these neurotropic viral diseases development of more effective intervention and antiviral treatment regimens is an urgent need.

[Bangladesh Journal of Infectious Diseases 2016;3(1):1-2]

[Citation: Hussain ME. Neurotropic Viral Infections in Bangladesh: Burden and Challenges. Bangladesh J Infect Dis 2016;3(1):1-2]

\section{References}

1. Noor R, Munna MS. Emerging diseases in Bangladesh: current microbiological research perspective. Tzu Chi Med J 2015;27(2):49-53.

2. Uddin PKMM, Morshed AMA, Fatema K, Nisa MJ, Das P, et al. Prevalence of Herpes Simplex Virus Infection among Adults Citizens of Dhaka, Bangladesh. J Blood Disord Transfus 2015;S5:003

3. Mamun SA, Chowdhury MAH, Khan RM, Sikder MAU, Hoque MM. Herpes Simplex Virus (HSV) Infection in Men with Genital Ulcer Disease (GUD) as Observed in Dhaka Medical College Hospital. Med Today 2010;22(2):55-61

4. Institute of Epidemiology Disease Control \& Research. At the Front Line of Public Health; Five Year Report from 20062011. WHO country Office; Web address: http://www.iedcr.gov.bd/index.php/publications/item/hospitalbased-influenza-copy

5. Gibney L, Macaluso M, Kirk K, Hassan MS, Schwebke J, Vermund $\mathrm{SH}$, et al. Prevalence of infectious diseases in Bangladeshi women living adjacent to a truck stand: HIV/STD/hepatitis/genital tract infections. Sexually Transmitted Infect 2001;77(5):344-50

6. Qutub M, Akhter J. Epidemiology of genital herpes (HSV-2) among brothel based female sex workers in Bangladesh. European J Epidemiol 2003;18(9):903-5

7. Ludlow M, Kortekaas J, Herden C, Hoffmann B, Tappe D, Trebst C, et al. Neurotropic virus infections as the cause of immediate and delayed neuropathology. Acta Neuropathologica 2016;131(2):159-84

8. Hemachudha T, Ugolini G, Wacharapluesadee S, Sungkarat W, Shuangshoti S, Laothamatas J. Human rabies: neuropathogenesis, diagnosis, and management. Lancet Neurol 2013;12:498-513

9. Chow FC, Glaser CA, Sheriff H, Xia D, Messenger S, Whitley R, Venkatesan A. Use of clinical and neuroimaging characteristics to distinguish temporal lobe herpes simplex encephalitis from its mimics. Clin Infect Dis 2015;60:13771383 